\title{
Ultra-wideband generator of coherent multicarriers for multipactor sensor
}

\section{system}

\author{
Xiaokang $\mathbf{Q i}^{1}$ and Huan $\mathbf{L i}^{2}$, a)
}

\begin{abstract}
Multicarrier signals are used in many radio frequency and microwave systems. A typical example is the wideband communication satellites. However, it is rare to require the carriers in such multicarrier systems to be coherent due to the fact that their frequencies are different. An exception is the testing system for Multipactor Sensor System (MSS). Compared with existing methods that rely on the heavy use of expensive microwave instruments, in this work, we demonstrate an ultra-wideband coherent multicarrier generator (UCMG) with commercial chips and economic devices, which works from UHF to Ku band. MSS experimental results verified the novelty and effectiveness of the proposed approach. The implemented UCMG would make it affordable for a regular microwave laboratory to conduct quantitative experimental researches on wideband multicarrier multipactors. It can also be widely used in other RF systems such as massive MIMO and large-scale phase array systems.

Keywords: coherent multicarrier generator, multipactors sensor system, FPLLs

Classification: Microwave and millimeter-wave devices, circuits, and modules
\end{abstract}

\section{Introduction}

For an electrical system, the word "coherence" implies that the phase differences between two (or more) sinusoidal signals remain constant. Conventionally, this coherence is defined for signals with the same frequency. Therefore, it is quite rare to require the carriers in a multicarrier system to be coherent, in which case their frequencies are different. For sinusoidal signals with different frequencies, "coherence" implies that the initial phases of such signals are not only constant, but also predefined. A typical example of a system requiring such an unconventional coherence is the wideband multicarrier Multipactors Sensor System (MSS).

In 1934 [1] the multipactor phenomenon was observed in vacuum high-power microwave devices. After that, theory model and production of multipactor have acquired many results to throw light on the multipactor phenomenon [2, $3,4,5,6,7,8,9]$. Numercial and simulation method are introduced to find out multipactor effects in high-power microwave devices $[10,11,12,13,14,15,16,17,18,19,20]$. These conclusions show that it occurs when electrons ac-

${ }^{1}$ The Laboratory of Applied Research on Electromagnetics (ARE), Zhejiang University, Hangzhou 310027, China

2 Ocean Electronics Engineering and Intelligent System Institution, Zhejiang University, Zhoushan 316021, China

a)xdlee07@163.com

DOI: $10.1587 /$ elex.17.20200283

Received August 20, 2020

Accepted August 26, 2020

Publicized September 7, 2020

Copyedited September 25, 2020 celerated by intense electric fields are self-sustained via an avalanche due to the secondary electron emission. In a multipactor, secondary electrons can be exponentially increased. The resultant convection current can severely interfere with useful signals, or even damage the device [3]. Therefore, the prevention of multipactor discharges is of vital importance for high-power microwave systems in space applications. With the rapid development of multicarrier high-power systems such as wideband communication satellites, researches on MSS become more and more important [6, 7, 21, 22]. In order to investigate the occurrence threshold of multicarrier multipactor, ultra-wideband generation of coherent multicarrier signals is indispensable. Unfortunately, only a few works were involved with experimental setups of MSS for the multicarrier multipactor thresholds measurement $[7,21,22,23,24,25]$ until recently. Apart from a narrow-band UHF system, the only wideband systems are the 12-channel $\mathrm{Ku}$-band MSS established with the massive use of expensive commercial instruments [7, 23, 25] which is hard to afford for regular laboratories.

In this work, we demonstrate the experimental implementation of an ultra-wideband coherent multicarrier generator (UCMG) based on a state-of-the-art Fractional-N phaselocked loops technique. Compared with existing methods that rely on the heavy use of expensive microwave instruments, proposed 12-channel UCMG is implemented with commercial chips and economic devices. It works with an ultra-wide bandwidth from UHF to Ku band. Experimental results verified the novelty and effectiveness of the proposed approach. The implemented UCMG would make it affordable for a regular microwave laboratory to setup an experimental MSS and conduct quantitative researches on wideband multicarrier multipactors. It can also be widely used in various systems such as massive MIMO and largescale phase array systems.

The paper is arranged as follow. In section 2, the implementation of a MSS and the generation of coherent multicarrier from proposed UCMG is described. In section 3 , the principle and implementation of the UCMG is clearly introduced. In section 4, the in-system calibration of UCMG and its usage in the MSS is demonstrated. In section 5, we draw a brief conclusion of this paper.

\section{System description}

In Figure 1, a block diagram of a convesional MSS is introduced, which consists of three subsystems, i.e., a high-power 


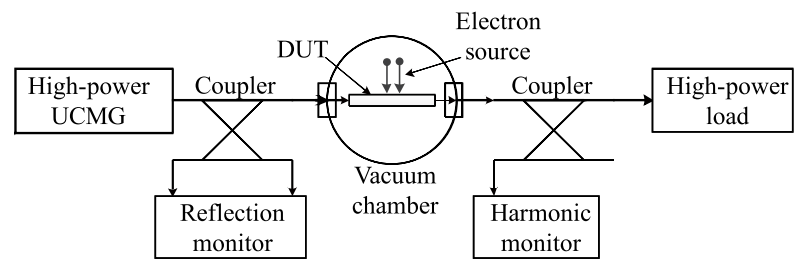

Fig. 1 Experimental setup of conventional MSS

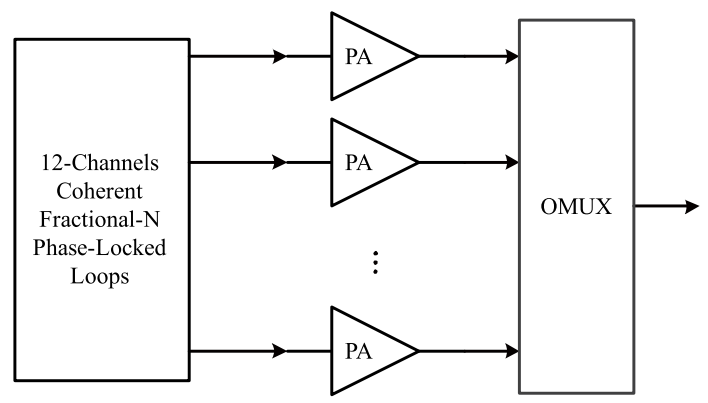

Fig. 2 Block diagram of the high-power UCMG

UCMG, a reflection monitor, and a vacuum chamber. An additional harmonics monitor is needed in order to observe the nonlinear response of the device under test (DUT) producing in the MSS [26]. At first, the UCMG will generate a coherent multicarrier signal to excite the DUTs. As the power of the multicarrier signal increasing, a sudden change of the multicarrier signal refection will be observed from the reflection monitor. So, the amplitude of input multicarrier power that cause this change can be regarded as the multipactor threshold of the DUT.

The block diagram of proposed UCMG is shown in Figure 2. It includes 12-channel vector RF signal generators, the power management module and an output multiplexor (OMUX) that is used to combine multiple carriers into a coherent multicarrier signal.

In mathematics, the multicarrier signal $S(t)$ can be written as:

$$
S(t)=\sum_{i=1}^{N} A_{i} \cdot \cos \left(2 \pi\left(f_{1}+\sum_{j=1}^{i-1} \Delta f_{j}\right) \cdot t+\frac{\pi}{180} \cdot \varphi_{i}\right)
$$

where $N$ is the number of carriers, $A_{i}$ and $\varphi_{i}$ are the amplitude and phase of the $i$-th carrier, respectively. $f_{1}$ is the frequency of the first carrier, and $\Delta f_{j}$ means the frequency spacing between two close carriers. $S(t)$ is periodic, whose periodicity is the least common multiple of the periods of all carriers.

In practical systems, $A_{i}$ and $\Delta f_{j}$ are normally constants, thus the waveform of $S(t)$ is only determined by $\varphi_{i}$. So, in the "in-phase" case where $\varphi_{i}$ is a constant [7], equation (1) can be re-written as a sinc function:

$$
S(t)=A_{0} \frac{\sin (N \pi \Delta f t)}{\sin (\pi \Delta f t)} \cdot \cos \left[2 \pi\left(f_{1}+\frac{N-1}{2} \Delta f\right) \cdot t\right]
$$

And in the "triangular-phase" case where the initial phases of the $\mathrm{N}$ carriers have a discrete linear relationship [7], the waveform of $S(t)$ can be described as:

$$
S(t)=A_{0} \sum_{i=1}^{\frac{N-1}{2}} \cos \left\{2 \pi\left[f_{1}+(i-1)\right] \cdot t\right.
$$

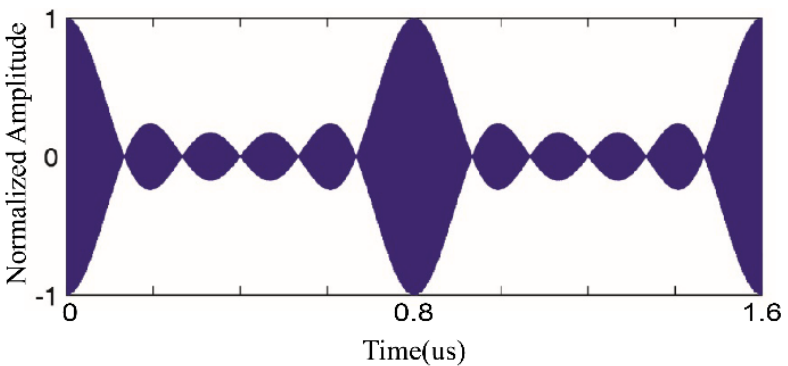

(a)

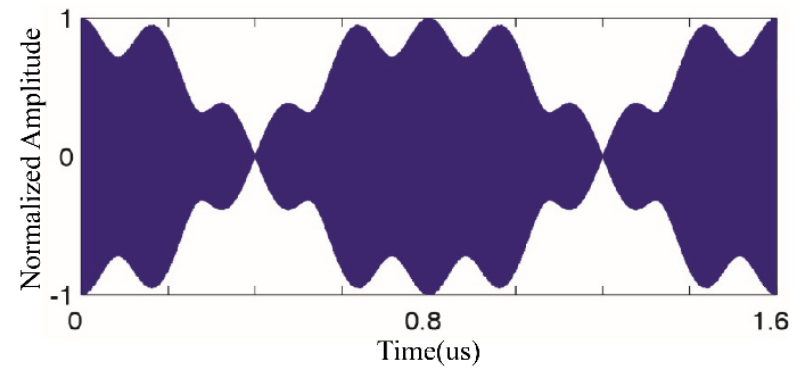

(b)

Fig. 3 Waveform of 6-carrier signal. (a) "in-phase" 6-carier signal. (b) "triangle-phase" 6-carrier signal.

$$
\begin{array}{r}
\left.+\frac{\pi}{180} \cdot \frac{4 k(N-2)(i-1)}{(N-1)^{2}}\right\} \\
+A_{0} \sum_{i=\frac{N+1}{2}}^{N} \cos \left\{2 \pi\left[f_{1}+(j-1) \cdot \Delta f\right] \cdot t\right. \\
\left.+\frac{\pi}{180} \cdot \frac{4 k(N-2)(N-i)}{(N-1)^{2}}\right\}
\end{array}
$$

For demonstration, Figure 3 shows the waveform of 2 sixcarrier signal as (2) and (3) described, where $f_{1}=800 \mathrm{MHz}$ and $\Delta f_{j}=1.25 \mathrm{MHz}$. Figure 3 indicates that with the same $f_{1}$ and $\Delta f_{j}$, different phase combinations can result in very different waveforms. Also, the multipactor thresholds of the same DUT can be significantly different.

That is the reason why the UCMG used in an MSS should be able to set the phases, frequencies and amplitudes of each carriers independently. However, because of the strict demand for the independence of each carrier's initial phase, amplitude and frequency, the generation of exciting signal of a MSS is of great challenge for regular microwave laboratory who wants to implement an experimental setup of a MSS.

To our knowledge, expensive commercial vector RF signal generators are of great essentials for current MSS testing systems. In [25], 12 synchronized commercial RF signal generators and 12 phase shifters were used to build a MSS testing system. In [7], a Ku-band multicarrier generator was implemented based on 12 L-band vector signal generators and $12 \mathrm{Ku}$-band up-converters. But for us, all these difficulties can be solved with the UCMG proposed in this paper. The technique details of the PLLs based CMS is clearly introduced in next section.

\section{Implementation of the UCMG}

\subsection{Principle}

As a very important component for signal sources imple- 


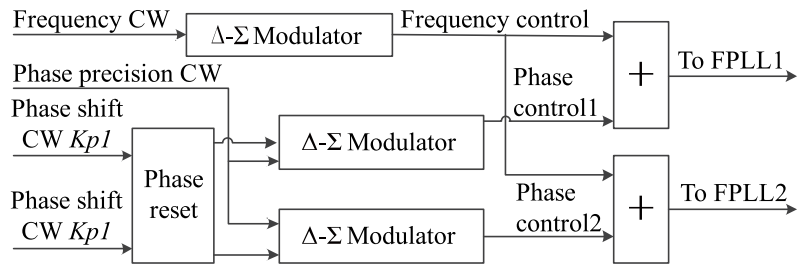

Fig. 4 Principle of new Fractional-N PLL

mentation, Fractional-N PLLs have been widely used to generate RF signals of many devices or microwave circuits [27, 28, 29, 30, 31]. However, limited with the design of PLL architecture, the output signals' frequency and phase of traditional Fractional-N PLLs are related parameters, which cannot be controlled as user's demand independently [32]. Recent studies solved this problem by introducing a cyclically shifting, the control word of frequency divider to a Fractional-N PLL. This improvement makes conventional PLLs can assign a certain value as the initial phase of the output RF signal, even if the output signal is predefined at different frequency. This is the technique that we use to implement the proposed UCMG.

As Fig. 4 shows that the phase control action is related with four control words $(\mathrm{CW})$, i.e., the frequency $\mathrm{CW}$, phase precision $\mathrm{CW}$, and two phase-shift CWs (Kpl and $K p 2)$. Firstly, $K p 1$ and $K p 2$ are sent to phase reset register. Then, they are written into delta-sigma modulators together with the phase CW. Finally, the modulated results of phase shift CWs and phase CW are added with the shifted frequency CWs respectively. Now, these frequency dividers (FDs) are worked out and written into the FD registers. According to [33], the frequency and phase shift control can be mathematically described by (4) and (5).

$$
\begin{gathered}
f_{0}=f_{r} \cdot\left\{\left[N_{f}+\left(K_{f}-1\right) / M\right]+1 / M\right\} \\
\Delta \varphi=2 \pi \cdot\left(K_{p 1}-K_{p 2}\right) / M
\end{gathered}
$$

where $f_{r}$ is the reference frequency, $f_{o}$ is the frequency of $V C O . N_{f}, K_{f}$, and $M$ are the frequency and phase precision CWs, respectively. $\Delta \varphi$ is the phase difference between the outputs of the Fractional-N PLLs.

As a result, the phase difference $\Delta \varphi$ is only related with CWs $K_{p 1}, K_{p 2}$ and $M$. When $M$ is a constant, $K_{p 1}$ and $K_{p 2}$ can determine the phase difference, i.e. $\Delta \varphi$. According to (5), the phase control precision can be improved as needed by increasing the width of $M$ theoretically. With the technique of programmable device and micro-processor, digital bitwidth is no longer a limitation in digital control unit design, which can always have sufficient bit-width to meet system's requirements. Therefore, this technique can be well used to solve the difficulty in implementing the UCMG.

\subsection{Implementation of UCMG}

Figure 5 shows the implementation of the Fractional-N PLLs based UCMG. It consists of 12 channels of Fractional-N PLL based signal generator, one clock management module designed with a TCXO, one digital control interface and a configure software. As Figure 5 indicates, each channel of Fractional-N PLL signal generator includes a PLL module to generate assigned RF signal, a filter network to cancel the



Fig. 5 Details of UCMG architecture

noise and harmonic waves and a power management module to control the amplitude of RF signal's output power. The clock management module is designed to synchronize 12 channels' reference clock by dividing one input $10 \mathrm{MHz}$ clock signal into 12 output clock signals, to make sure all 12 channels' output signal are coherent. In the digital control interface module, a pair of programmable MCU and CPLD are used transfer control words from configure software to Fractional-N PLL registers, acting as communication interface between control software and the UCMG.

In this paper, the Fractional-N PLLs were a commercial chip from ADI named ADF5355. Within a 24-bit CW in register 3 , the phase of the RF output frequency can adjust in 24-bit steps, from $0^{\circ}(0)-360^{\circ}\left(2^{24}-1\right)$. In order to minimize the phase noise and jitter, we choose a high-stability TCXO from RAKON named RTX7050A to act as references clock of all 12 channels Fractional-N PLLs. In order to make sure all Fractional-N PLLs to be initialized at the same time, digital control interfaces designed with CPLD is used to trigger the locking action synchronously. As Figure 6 shows, all Fractional-N PLLs are fixed on the digital control interface module, where they can get power supply, control words, reference clock. To make the hardware details and chips selection more clear, the main commercial components used in this UCMG are listed in Table I.

With the design of the filter network and RF signal path, the UCMG can work in an ultra-wide frequency range from $300 \mathrm{MHz}-18 \mathrm{GHz}$. Figure 7 shows the frequency spectrum of one Fractional-N PLL signal generator who works at $16 \mathrm{GHz}$. The used real-time spectrum analyzer is Agilent E4407B. From the data of spectrum analyzer, we can calculate the phase noise of the $16 \mathrm{GHz}$ sinusoidal signal output from one channel of the proposed UCMG with equation (6) [34].

$L(f)=P_{n}(\mathrm{dBm} / \mathrm{Hz})-P_{s}(\mathrm{dBm})-10 \log (1.1 \times R E S . B W)$

Where, $P_{n}$ indicates the noise power in a $1 \mathrm{~Hz}$ bandwidth, and $P_{S}$ indicates total signal power in test bandwidth. The calculated result shows that the phase noise of proposed $\mathrm{UCMG}$ is $-75 \mathrm{dBc} / \mathrm{Hz}$ @ $1 \mathrm{kHz},-82 \mathrm{dBc} / \mathrm{Hz} @ 10 \mathrm{kHz}$, $-84 \mathrm{dBc} / \mathrm{Hz} @ 20 \mathrm{kHz}$, which is satisfactory for the strict requirement of a MSS. Since the waveform jitter is mainly related with the phase noise of each carrier, we can draw the conclusion that multicarrier signal combined with proposed UCMG would be very stable. 

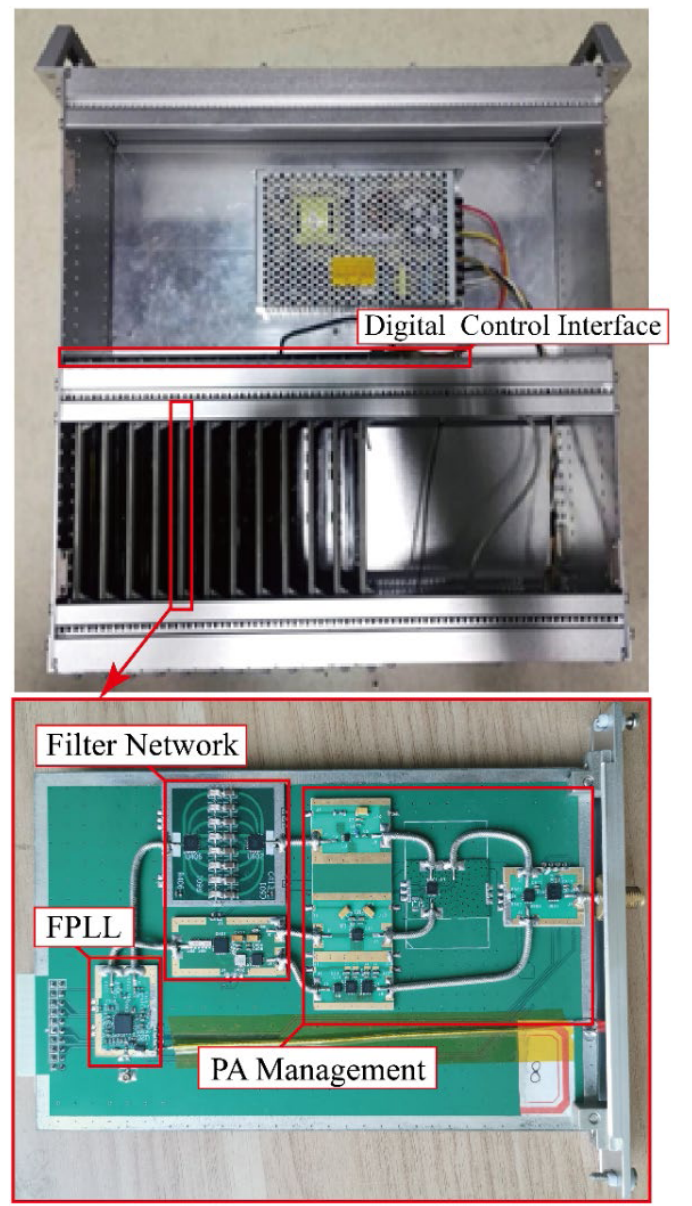

Fig. 6 Fractional-N PLLs based UCMG

Table I Key Components Used in the UCMG

\begin{tabular}{c|c|c}
\hline Device & Manufacture & Function \\
\hline ADF5355 & ADI & PLL \\
\hline STM8AF6286 & ST & Microprocessor \\
\hline EPM570T144C4 & ALTERA & CPLD \\
\hline RTX7050A & RAKON & TCXO \\
\hline HMC573 & ADI & Frequency Multiplier \\
\hline HMC311 & ADI & Low-Noise-Amplifier \\
\hline HMC3653 & ADI & Low-Noise-Amplifier \\
\hline HMC451 & ADI & Low-Noise-Amplifier \\
\hline HMC1019 & ADI & Attenuator \\
\hline
\end{tabular}

\section{Result and in-system calibration}

Figure 8 shows the experimental setup of the testing of the implemented UCMG, of which six channels are assigned to output a serious of 6-carriers signal in "in-phase" mode, the other six channels are assigned to output a serious of 6-carriers signal in "triangle-phase" mode. Two combined coherent multicarrier signals were sent to a wideband oscilloscope with $2.5 \mathrm{GS} / \mathrm{s}$ sampling rate. The frequency of the first carrier is set as $800 \mathrm{MHz}$, and $\Delta f_{j}$ is set as $1.25 \mathrm{MHz}$.

One thing that has to be mentioned is that, even though we design and assemble each channel of Fractional-N PLL signal generator all the same. The phase delay and amplitude between different channels are still can hardly be unbalanced, which will result in waveform distortions of the combined signals inevitably. Now, the advantage of pro-

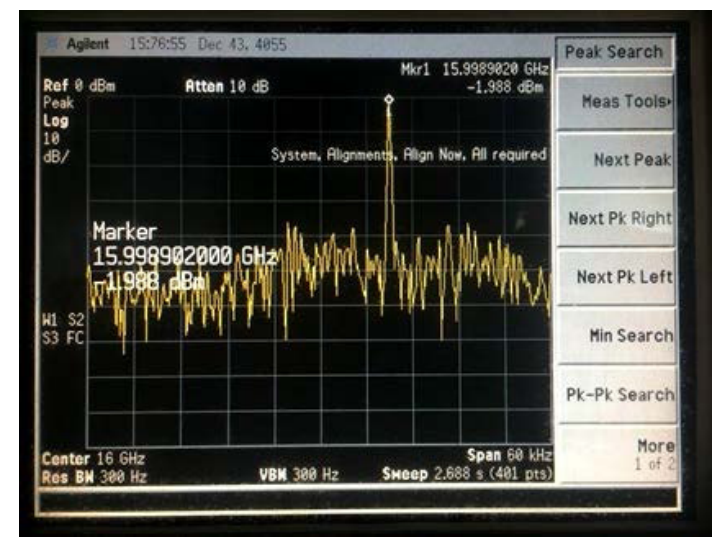

Fig. 7 Frequency spectrum of FPLL

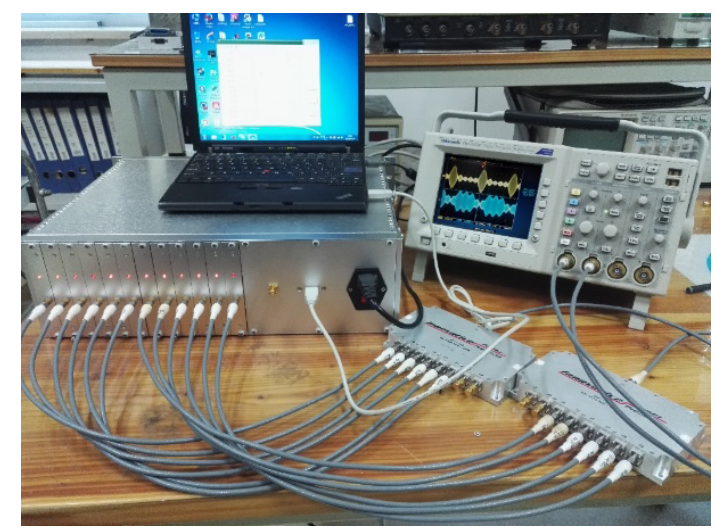

Fig. 8 Experimental setup of the proposed UCMG

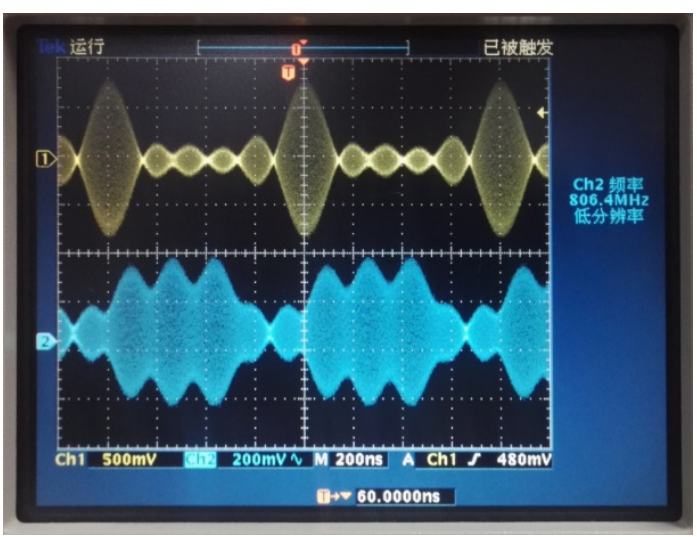

Fig. 9 Waveforms of the 6-carrier "in-phase" and "triangle-phase" signals

posed UCMG in channel phase adjustment can be useful to solve this problem with a three-step in-system calibration.

Firstly, the output multicarrier signal of UCMG will be sampled with a wideband instrument, like the oscilloscope we use in this experiment. Secondly, we use an inverse Fourier transform of the sampled data to acquire the phase and amplitude of each channel, and the phase and amplitude error among each channel can be retrieved by comparing them with the predefined ones. Finally, a negative feedback mechanism is introduced to calibrate the unbalanced phase delay and amplitude among different channels.

Figure 9 shows the photographs of the 6-carrier "in-phase" and "triangle-phase" multicarrier signals output from the 12-channel UCMG after such an in-system calibration. The 


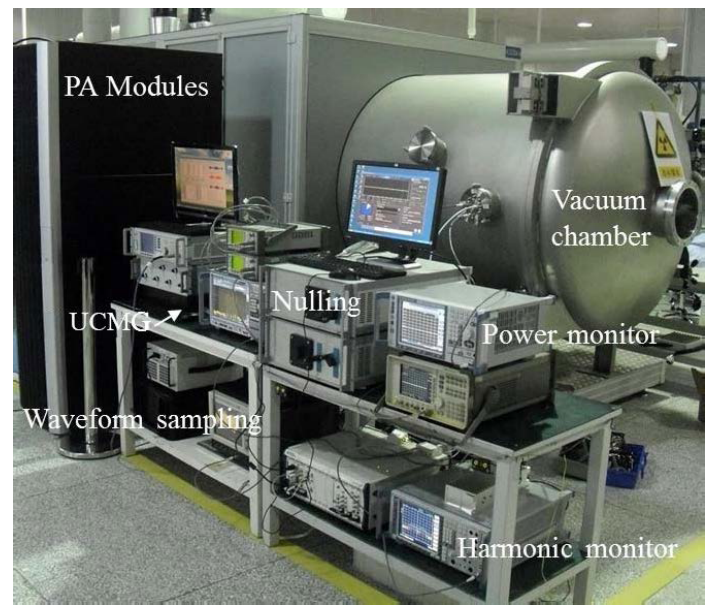

Fig. 10 An example of MSS setup with proposed UCMG

experiment result shows that these signals are nearly the same with the waveform shown in Figure 3, which have the same carrier configuration as the experimental setup.

In Figure 10, an example of MSS experimental setup based on proposed UCMG is demonstrated, according to implementation in Figure 1. Compared with current methods that use a serious of expensive microwave instruments to setup MSS, the UCMG proposed in this paper is compact, low-cost, and satisfactory for experimental usage. It makes MSS related research an affordable field for ordinary microwave laboratory. What's more, it can also bring significant convenience and quantitative measurement of multipator threshold for any multicarrier waveforms, with the functions of digital control system and in-system calibration technique.

\section{Conclusions}

As the most important and complex part of a conventional MSS, we demonstrate a novel, cost-effective approach to design a 12-channel ultra-wideband coherent multicarrier generator for the experimental setup of MSS. With design of the state-of-the-art Fractional-N PLLs system and in-system calibration techniques, the UCMG can take the place of MSS's complex coherent multicarrier generation system which requires numbers of RF vector generator and a synchronize clock. What's more, the UCMG can work from UHF to KU band with each carrier's phases, frequencies and amplitudes predefined and adjusted independently. It can act as an experimental instrument for many microwave laboratories because of its economy, wide-band feature and satisfactory performance. It will help to promote in-depth experimental research on the multipactor threshold measurement and related area.

\section{Acknowledgments}

This work was supported by the NSFC under grants 61701437.

\section{References}

[1] P.T. Farnsworth: "Television by electron image scanning," J. Franklin
Inst. 218 (1934) 411 (DOI: 10.1016/S0016-0032(34)90415-4).

[2] J.R.M. Vaughan: "Multipactor," IEEE Trans. Electron Devices 35 (1988) 1172 (DOI: 10.1109/16.3387).

[3] L.-K. Ang et al.: "Power deposited on a dielectric by multipactor," IEEE Trans. Plasma Sci. 26 (1998) 290 (DOI: 10.1109/27.700756).

[4] M. Siddiqi and R. Kishek: "Map-based multipactor theory for crossfield devices," IEEE Trans. Electron Devices 66 (2019) 3162 (DOI: 10.1109/TED.2019.2914343).

[5] S. Anza, et al.: "Multipactor theory for multicarrier signals," Phys. Plasmas 18 (2011) 032105 (DOI: 10.1063/1.3561821).

[6] S. Anza, et al.: "Long-term multipactor discharge in multicarrier systems," Phys. Plasmas 14 (2007) 082112 (DOI: 10.1063/1.2768019).

[7] S. Anza, et al.: "Prediction of multipactor breakdown for multicarrier applications: the quasi-stationary method," IEEE Trans. Microw. Theory Techn. 60 (2012) 2093 (DOI: 10.1109/TMTT.2012.2197021).

[8] A. Valfells, et al.: "Space-charge effects on multipactor on a dielectric," IEEE Trans. Plasma Sci. 28 (2000) 529 (DOI: 10.1109/ 27.887665).

[9] S. Riyopoulos, et al.: "Effect of random secondary delay times and emission velocities in electron multipactors," IEEE Trans. Electron Devices 44 (1997) 489 (DOI: 10.1109/16.556160).

[10] J.W. You, et al.: "Highly efficient and adaptive numerical scheme to analyze multipactor in waveguide devices," IEEE Trans. Electron Devices 62 (2015) 1327 (DOI: 10.1109/TED.2015.2399360).

[11] Y. Li, et al.: "Enhanced dynamics simulation and threshold analysis of multipaction in the ferrite microwave component," Physics of Plasmas 24 (2017) 023505 (DOI: 10.1063/1.4975190).

[12] Y. Li, et al.: "Three-dimensional numerical simulation of multipactor in open antenna structures," Microwave Conference Proceedings (APMC) 2013 Asia-Pacific (2013) 1058 (DOI: 10.1109/ APMC.2013.6695077).

[13] G. Cheng, et al.: "Monte Carlo study of the single-surface multipactor electron discharge on a dielectric," IEEE Trans. Plasma Sci. 37 (2009) 1968 (DOI: 10.1109/TPS.2009.2016967).

[14] V. Semenov, et al.: "Multipactor in microwave transmission systems using quadrature phase-shift keying,” IEEE Trans. Plasma Sci. 38 (2010) 915 (DOI: 10.1109/TPS.2010.2040610).

[15] D. González-Iglesias, et al.: "Experimental analysis of the multipactor effect with RF pulsed signals," IEEE Electron Device Lett. 36 (2015) 1085 (DOI: 10.1109/LED.2015.2468068).

[16] S.V. Langellotti, et al.: "CST particle studio simulations of coaxial multipactor and comparison with experiments," IEEE Trans. Plasma Sci. 48 (2020) 1942 (DOI: 10.1109/TPS.2020.2981257).

[17] X. Zhang, et al:: "Multipactor analysis in circular waveguides excited by $\mathrm{TM}_{01}$ mode," IEEE Trans. Electron Devices 66 (2019) 4943 (DOI: 10.1109/TED.2019.2941594).

[18] Z. Zhang, et al.: "An analytical model of one-sided multipactor on a dielectric of a metal surface for spacecraft application," IEEE Trans. Electron Devices 66 (2019) 4921 (DOI: 10.1109/TED 2019.2937752)

[19] M.K. Joshi, et al.: "Multiphysics and multipactor analyses of TE022Mode high-power X-band RF window," IEEE Microwa. Wireless Compon. Lett. 30 (2020) 272 (DOI: 10.1109/LMWC.2020.2971652).

[20] D. González-Iglesias, et al.: "Analysis of multipactor RF breakdown in a waveguide containing a transversely magnetized ferrite," IEEE Trans. Electron Devices 63 (2016) 4939 (DOI: 10.1109/TED. 2016.2614370).

[21] X. Wang, et al.: "Generation of coherent multicarrier signals for the measurement of multicarrier multipactor," IEEE Trans. Instrum. Meas. 66 (2017) 3357 (DOI: 10.1109/TIM.2017.2746381).

[22] X. Wang, et al:: "Monte Carlo analysis of occurrence thresholds of multicarrier multipactors," IEEE Trans. Microw. Theory Techn. 65 (2017) 2734 (DOI: 10.1109/TMTT.2017.2661744).

[23] A. Berenguer, et al.: "Experimental study of the multipactor effect in a partially dielectric-loaded rectangular waveguide," IEEE Microw. Wireless Compon. Lett. 29 (2019) 595 (DOI: 10.1109/LMWC. 2019.2929488).

[24] R.I. Cameron and M. Yu: "Design of manifold-coupled multiplexers," IEEE Microw. Mag. 8 (2007) 46 (DOI: 10.1109/MMM.2007. 904715).

[25] D. Wolk and D. Rosowsky: "Design and high power test of a 12 
GHz/12 channel contiguous output multiplexer," Proc. 14th Int. Commun. Satellite Syst. Conf. (1992) 943 (DOI: 10.2514/6.1992-1934).

[26] ECSS-E20-01A (2003).

[27] D. Banerjee: PLL Performance, Simulation, and Design (Dog Ear Publishing, Indianapolis, IN, 2006) 4th ed.

[28] H. Zhou, et al.: "A monolithic sub-sampling PLL based 6-18 GHz frequency synthesizer for C, X, Ku band communication," ICE Trans. Electron. 98 (2015) 16 (DOI: 10.1587/transele.E98.C.16).

[29] W. Zou, et al.: "A wideband low-jitter PLL with an optimized Ring-VCO,” IEICE Electron. Express 17 (2020) 20190703 (DOI: 10.1587/elex.17.20190703).

[30] Z. Cai, et al.: "On-chip long-term jitter measurement for PLL based on undersampling technique," IEICE Electron. Express 10 (2013) 20130887 (DOI: 10.1587/elex.10.20130887).

[31] P. Qin, et al.: "A fast and efficient automatic frequency calibration technique for $10 \mathrm{GHz}$ PLLs," IEICE Electron. Express 11 (2014) 20140845 (DOI: 10.1587/elex.11.20140845).

[32] K. Tajima and R. Hayashi: "Novel phase difference control between output signals using fractional-N PLL synthesizers by cyclic shift of control data," IEEE MTT-S Int. Microw. Symp. Dig. (2007) 835 (DOI: 10.1109/MWSYM.2007.380089).

[33] K. Tajima, et al.: "Frequency and phase difference control using fractional-N PLL synthesizers by composition of control data," IEEE Trans. Microw. Theory Techn. 55 (2007) 2832 (DOI: 10.1109/TMTT. 2007.909149).

[34] Agilent Technologies: "Application note 1303 spectrum and signal analyzer measurements and noise," (2009). 